\title{
Association of lifetime exposure to fluoride and cognitive functions in Chinese children: A pilot study
}

\section{Citation}

Choi, Anna L., Ying Zhang, Guifan Sun, David C. Bellinger, Kanglin Wang, Xiao Jing Yang, Jin Shu Li, Quanmei Zheng, Yuanli Fu, and Philippe Grandjean. 2015. "Association of Lifetime Exposure to Fluoride and Cognitive Functions in Chinese Children: A Pilot Study." Neurotoxicology and Teratology 47 (January): 96-101. doi:10.1016/j.ntt.2014.11.001.

\section{Published Version}

10.1016/j.ntt.2014.11.001

\section{Permanent link}

http://nrs.harvard.edu/urn-3:HUL.InstRepos:37221747

\section{Terms of Use}

This article was downloaded from Harvard University's DASH repository, and is made available under the terms and conditions applicable to Open Access Policy Articles, as set forth at http:// nrs.harvard.edu/urn-3:HUL.InstRepos:dash.current.terms-of-use\#OAP

\section{Share Your Story}

The Harvard community has made this article openly available.

Please share how this access benefits you. Submit a story.

Accessibility 
Association of lifetime exposure to fluoride and cognitive functions in Chinese children: A pilot study

Anna L Choi ${ }^{\mathrm{a}, *}$, Ying Zhang ${ }^{\mathrm{b}}$, Guifan Sun ${ }^{\mathrm{c}}$, David Bellinger ${ }^{\mathrm{a}, \mathrm{d}}$, Kanglin Wang ${ }^{\mathrm{e}}$, Xiao Jing Yang ${ }^{\mathrm{f}}$, Jin Shu Lif ${ }^{\mathrm{f}}$, Quanmei Zheng ${ }^{\mathrm{c}}$, Yuanli Fu ${ }^{\mathrm{g}}$, Philippe Grandjean ${ }^{\mathrm{a}, \mathrm{h}}$

${ }^{a}$ Department of Environmental Health, Harvard School of Public Health, Boston, Massachusetts, USA

${ }^{\mathrm{b}}$ School of Stomatology, China Medical University, Shenyang, China

${ }^{\mathrm{c}}$ School of Public Health, China Medical University, Shenyang, China

${ }^{\mathrm{d}}$ Neurology, Children's Hospital, Boston, USA

${ }^{\mathrm{e}}$ Mianning Center for Disease Control and Prevention, Xichang, Sichuan, China

${ }^{\mathrm{f}}$ Sichuan Center for Disease Control and Prevention, Chengdu, Sichuan, China

${ }^{\mathrm{e}}$ Center for Disease Control and Prevention of Liangshan Perfecture, Xichang, Sichuan, China

${ }^{\text {h }}$ Institute of Public Health, University of Southern Denmark, Odense, Denmark

Address correspondence to:

Anna L Choi, Department of Environmental Health, Harvard School of Public Health, Landmark

Center 3E, 401 Park Dr., Boston, MA 02215, USA

Phone: +01617384 8646 Fax: +01 6173848994

E-mail: achoi@hsph.harvard.edu 


\begin{abstract}
Background: A systematic review and meta-analysis of published studies on developmental fluoride neurotoxicity supports the hypothesis that exposure to elevated concentrations of fluoride in water is neurotoxic during development.
\end{abstract}

Methods: We carried out a pilot study of 51 first-grade children in southern Sichuan, using the fluoride concentration in morning urine after usage of fluoride-free drinking water; fluoride in well-water source; and dental fluorosis status as indices of past fluoride exposure. We administered a battery of age-appropriate, relatively culture-independent tests that reflect different functional domains: the Wide Range Assessment of Memory and Learning (WRAML), Wechsler Intelligence Scale for Children-Revised (WISC-R) digit span and block design; finger tapping and grooved pegboard. Confounder-adjusted associations between exposure indicators and test scores were assessed using multiple regression models.

Results: Dental fluorosis score was the exposure indicator that had the strongest association with the outcome deficits, and the WISC-R digit span subtest appeared to be the most sensitive outcome, where. moderate and severe fluorosis was associated with digit span total score difference of $-4.28(95 \%$ CI $-8.22,-0.33)$ and backward score with -2.13 (95\% CI -4.24, -0.02).

Conclusions: This pilot study in a community with stable lifetime fluoride exposures, supports the notion that fluoride in drinking water may produce developmental neurotoxicity, and that the dose-dependence underlying this relationship needs to be characterized in detail.

\title{
Keywords
}

Children; Confounding; Fluoride; Fluorosis; Neuropsychological measures; Prenatal exposure 
delayed effects

Abbreviations

CDC, Center for Disease Control

SD, Standard deviation 


\section{Introduction}

The developing human brain is much more susceptible to injury caused by toxicants than is the mature brain, and the damage incurred is likely to be of a permanent nature as the major windows of developmental vulnerability occur in utero and during infancy and early childhood (Grandjean and Landrigan, 2006; Rice and Barone, 2000). Chemicals can, therefore, cause permanent brain injury at low levels of exposure that would have little or no adverse effect in an adult (Grandjean and Landrigan, 2014).

Fluoride occurs naturally in the environment from the weathering of fluoride-containing rocks and soils, and leaching from soil into groundwater. Fluoride is also released into the environment via coal combustion and other industrial sources, either by direct deposition or by deposition to soil and subsequent runoff into water (NRC 2006; WHO 2002). The major sources of human exposure to fluoride are drinking water, food, dental products, and pesticides (NRC 2006). Fluoride is a trace element that is necessary for the human body. A proper amount of fluoride not only prevents dental caries, but also promotes the use of calcium and phosphorus and the calcium sediment in the bone, stimulates bone growth and maintains bone health (Dean and Elvove 1936, WHO 1958).

However, the developing human brain may be exposed prenatally to fluoride as fluoride readily crosses the placenta (ATSDR 2003). In laboratory studies, the central nervous system (CNS) may be vulnerable to fluoride. Fluoride accumulates in brain tissues and may affect the hippocampus, the central processor of memory, in learning and memory functions (Mullenix et al. 1995; Chioca et al. 2008). Potential effects on the neurodevelopment in children have been explored mainly in China where many urban and rural communities are located in endemic 
fluorosis areas. Thus, a National Research Council report reviewed the effects of fluoride in drinking water on human health, including the cognitive capacities in children (NRC 2006). Many relevant studies were identified that, collectively, supported the hypothesis that fluoride is a developmental neurotoxicant, but because many of the studies did not provide sufficient information about the neurobehavioral tests used and the testing conditions, the report recommended that additional, more rigorous research be conducted.

In response to this report, the U.S. Department of Health and Human Services (DHHS) is proposing to set the recommended level of fluoride in drinking water at $0.7 \mathrm{mg} / \mathrm{L}$, the lower end of the current range of 0.7 to $1.2 \mathrm{mg} / \mathrm{L}$, and the U.S. Environmental Protection Agency (U.S. EPA) is considering lowering the maximum amount of fluoride allowed in drinking water, which is currently $4 \mathrm{mg} / \mathrm{L}$ (U.S. EPA 2011).

To investigate the effects of increased fluoride exposure on children's neurobehavioral development, we performed a systematic review and meta-analysis of 27 cross-sectional studies of children exposed to fluoride in drinking water, mainly from China (Choi et al. 2012). Our results showed that the standardized weighted mean difference (SMD) in IQ score between exposed and reference populations across studies that gave the average difference in standard deviations (SDs) was -0.45 (95\% confidence interval: $-0.56,-0.36)$. For commonly used IQ scores with a mean of 100 and a SD of $15,0.45 \mathrm{SDs}$ is equivalent to $6 / 75$ points (rounded to 7 points). The results therefore showed an average IQ decrement of about seven points in children with increased fluoride exposure. The elevated exposure groups had access to drinking water with fluoride concentrations ranging from $0.57 \mathrm{mg} / \mathrm{L}$ to $11.5 \mathrm{mg} / \mathrm{L}$. As the average difference in terms of IQ between elevated and background exposure groups corresponded to approximately 7 
points, our review highlighted a need to further characterize the dose-response association including improving assessment and control of potential confounders.

The current pilot study was undertaken to assess the feasibility of administering a comprehensive battery of neurodevelopmental tests to a population of school children in China in order to test the hypothesis that increased fluoride exposure is related to impairments in neurobehavioral development. Specifically, we identified a population of children who had been exposed to stable fluoride concentrations in drinking water since conception and assessed the feasibility and validity of exposure assessment and neurobehavioral testing under field conditions in rural China. In this country, a country-wide effort to provide microbiologically safe drinking-water in rural communities secured piped spring water or well-water for each household. Families residing at the same location could therefore be characterized in regard to fluoride exposure based on the concentration of fluoride in a child's water source.

\section{Materials and methods}

\subsection{Study population}

We carried out a field study of 51 first-grade children, aged 6-8 years in 2011, who resided in Mianning County in southern Sichuan, China. While there is a wide range of fluoride concentrations in drinking water in this area, the residence-specific water sources have very stable fluoride levels. For families remaining at the same residence, the children have therefore been exposed to a stable fluoride concentration since conception. Children who did not speak Chinese, who were not students at the Primary School of Sunshui Village in Mianning County, or who had a chronic or acute disease that might affect neurobehavioral function tests were 
excluded.

The study protocol was approved by the Ethics Committee of China Medical University and by the Institutional Review Board at the Harvard School of Public Health. Written informed consent was obtained from all parents or guardians.

\subsection{Measurements of Exposure}

Fluoride concentrations in well-water in the communities were measured and recorded by Mianning County Center for Disease Control (CDC). Apart from seasonal changes, the wellwater fluoride concentrations have remained the same over the years, and the residents generally consistently use the same source for their drinking water needs. Well-water fluoride concentrations of the mother's residence during pregnancy and onwards were therefore used to characterize a child's lifetime exposure. Review of CDC records documented that levels of other contaminants, including arsenic and lead, are very low in the area.

Due to the stable residence of the study population, current exposures are thought to be representative of chronic exposures. Additionally, the urinary fluoride excretion after an exposure-free night was used as a reflection of the release from skeletal deposits of fluoride. To provide a measure of the accumulated body burden, each child was therefore given a $330 \mathrm{ml}$ (11.2 oz) bottle of Robust $\odot$ distilled water (free from fluoride and other contaminants) to drink the night before the clinical examinations, after emptying the bladder and before bedtime. The parents or guardians were instructed to ensure that the child would only drink the distilled water during this period and that the child did not have other sources of water prior to the study visit day. The first urine sample the following morning was collected at home, and the fluoride 
concentration was determined on a $5 \mathrm{~mL}$ sample using an ion-specific electrode (Whitford 1996) at the Mianning Center for Disease Control (CDC).

One of the investigators (YZ), a dentist, performed a blinded dental examination on each child's permanent teeth to rate the degree of dental fluorosis using the Dean Index (Dean 1942; WHO 1997). Dental fluorosis is caused by excess fluoride exposure when the teeth are being formed. The Dean Index is a commonly used index in epidemiological studies and remains the gold standard in the dentistry armamentarium. The Index has the following classification: Normal, Questionable, Very mild, Mild, Moderate, and Severe. The severity of the condition depends on the dose, duration, and the timing of fluoride intake. Questionable fluorosis indicates teeth with a few white flecks or spots. It is sometimes difficult to draw a clear distinction between the Questionable from the Normal. Very mild and mild forms of dental fluorosis indicate teeth have scattered white flecks, occasional white spots, frosty edges, or fine and lacy chalk-like lines. Moderate and severe forms of dental fluorosis indicate teeth have larger white spots and in the rare and severe form, rough and pitted surfaces.

The well-water fluoride concentrations of the residence during pregnancy and onwards, the fluoride concentration in a child's first urine sample in the morning, and the degree of the child's dental fluorosis were used as indicators exposure to fluoride.

A $20 \mu \mathrm{L}$ capillary blood sample was collected at the school by a Mianning CDC health practitioner and tested for possible iron deficiency which could be used as a covariate of neurodevelopmental performance. 


\subsection{Outcome measurements}

We adopted culture-independent tests considered feasible for children aged 6 to 8 years, and reflecting a variety of functional domains. The selection was based on several considerations. The very sparse literature on fluoride neurotoxicity does not provide strong clues as to what domains would be expected to be most affected. To date, studies have predominantly reported only on IQ scores (and often only on scores yielded by tests such as the Raven's Progressive Matrices test, which assesses a limited range of relevant functions). Therefore, we decided to use tests that assess various important domains, using those that we have found to be useful in other studies we have conducted in non-English speaking, rural populations, such as the Amazon and Ecuador (Grandjean et al. 1999, 2006).

The Wide Range Assessment of Memory and Learning (WRAML) (Sheslow and Adams, 1990) is used for the assessment of memory and learning. We used three subtests. The Finger Windows subtest assesses sequential visual memory. The Design Memory subtest assesses the ability to reproduce designs from memory following a brief exposure. The Visual Learning subtest assesses the ability to learn the locations of pictured objects over repeated exposures.

The Wechsler Intelligence Scale for Children-Revised (WISC-IV) included digit span for auditory span and working memory, and block design for visual organization and reasoning (Wechsler, 1974). Digit spans of increasing length were presented, and the children repeated strings of digits. Scores were total number of correct trials forward, backward, and total. For block designs, the children used red and white blocks to replicate stimulus designs presented on cards. Scoring was based on the WISC-R criteria combining a basic score for producing a correct design with bonus points for quick performances.

The Drawing subtest of the Wide Range Assessment of Visual Motor Ability (WRAVMA) 
(Adams and Sheslow, 1995) assesses the ability to copy, with a pencil, designs arranged in order of difficulty. One point is awarded for each possible item.

Finger tapping task is a measure of fine motor ability (Lezak, 1995). The children tapped a key for $15 \mathrm{sec}$ first with the preferred hand for practice, then twice with the preferred hand, and twice with the non-preferred hand. Scores consisted of the maximum number of taps in each condition.

The grooved pegboard test assesses manual dexterity (Knights and Moule, 1968). The children were presented a small board containing a $5 \times 5$ set of slotted holes angled in different directions and 25 pegs. They were required to insert the pegs into the holes as quickly as possible. The time to completion (in sec) for both dominant and non-dominant hand was recorded.

Such tasks have been used in diverse cultural settings as measures of brain functions (Grandjean et al., 1999; 2006). After translation and training, the examinations were conducted by experienced public health researchers and subsequently scored by a neuropsychologist (DB). Raw scores were used and age adjustment was performed in the statistical analyses.

\subsection{Measurement of covariates}

In this rural community, social differences are limited. The parents or guardians completed a questionnaire on demographic and personal characteristics including the child's sex, age at testing, parity, illnesses before age 3 , past medical history of the child and caretakers, parental or guardian age, education and occupational histories, and residential history, and household income. It is known that iron deficiency can impair motor and mental developments in children, iron concentration was therefore considered as a covariate. These potential confounders were used for adjustment in the statistical analysis. 


\subsection{Statistical analyses}

The distributions of fluoride concentrations in urine and water were skewed and were log (base 10) transformed to approximate a Gaussian distribution. For dental fluorosis, we combined the categories of the Dean Index into (1) normal/questionable; (2) very mild/mild; and (3) moderate/severe according to the clinical manifestations of dental fluorosis so that a sufficient number of subjects were in each category. The neurobehavioral test scores were normally distributed, although Block Design score only after squareroot transformation and pegboard time only after logarithmic transformation.

Associations of fluoride exposures with outcome parameters were determined using standard regression analysis with confounder adjustment. The corresponding 95\% confidence intervals (CI) were calculated.

To compare the associations between fluorosis and the neurobehavioral test scores, confounder-adjusted means were calculated for the three combined-categories of the Dean Index.

\section{Results}

Table 1 shows the characteristics of the 51 study children. There were slightly more girls than boys (53\% vs. 47\%), and the average age was 7.1 years. All subjects had birth weight more than $2500 \mathrm{~g}$. The hemoglobin and hematocrit levels were within normal ranges and did not suggest the presence of iron deficiency. Household income in the past year was less than 3000 yuan (US \$462 in 2011) for the majority of families (61\%), and most of the caretakers were farmers $(96 \%)$. 
The distributions of fluoride concentrations in urine and water are reported in Table 2. Urine fluoride was moderately correlated with water fluoride $(\mathrm{r}=0.66, \mathrm{p}<0.001)$. The permanent teeth of 8 children had not erupted, and their Dean Index, therefore, could not be determined and was treated as missing. Sixty percent of the subjects examined had moderate or severe fluorosis. These children were exposed to elevated fluoride concentrations in drinking water. Children with normal or questionable Dean index were all from households with a water fluoride concentration of $1 \mathrm{mg} / \mathrm{L}$ and had urinary fluoride excretion levels below $1 \mathrm{mg} / \mathrm{L}$.

The distributions of children's scores on the neuropsychological tests are shown in Table 3. Motivation to perform the tests is assumed to be optimal, and the test scores show sufficient dispersion to permit evaluation of their associations with fluoride exposure.

Results of multiple regression models show that moderate and severe fluorosis was significantly associated with lower total and backward digit span scores when compared to the reference combined categories of normal and questionable fluorosis (Table 4). Although the associations between fluoride in urine and in drinking water with digit span were not significant, they were in the anticipated direction. Motor coordination and dexterity were not significantly associated with fluoride in drinking water and fluorosis although higher levels were associated with poorer scores as well. Other outcomes did not reveal any association with the fluoride exposure.

Table 5 shows the adjusted means of the children's neuropsychological test scores by the Dean Index score. Children with moderate/severe fluorosis had significantly lower adjusted total and backward digit span mean scores when compared with those with normal/questionable fluorosis. Marginal significant mean differences in forward and total digit span scores were 
found between children with very mild/mild fluorosis and children with normal/questionable fluorosis.

\section{Discussion}

Results of our pilot study showed that moderate and severe dental fluorosis was significantly associated with deficits in WISC-R digit span. Children with moderate or severe dental fluorosis scored significantly lower in total and backward digit span tests than those with normal or questionable fluorosis. These results suggest a deficit in working memory. Scores on other tests did not show significant relationships with indices of fluoride exposure.

The 2006 National Research Council report recommended additional research on the effects of fluoride in drinking water on intelligence with different exposure concentrations, the use of neurobehavioral tests that measure reasoning ability, IQ, and memory, as well as the use of comparison groups similar to the groups with higher exposures in terms of culture and socioeconomic status (NRC 2006). Furthermore, our systematic review and meta-analysis of published studies supports the possibility of an adverse effect of high fluoride exposure on children's neurodevelopment, with suggestions for further characterization of the dose-response association (Choi et al., 2012).

A main purpose of this pilot project was to assess the feasibility of using the types of neurodevelopmental tests recommended in the NRC report in a population of children who had been exposed to a range of water fluoride concentrations since conception. Apart from seasonal changes, the drinking water fluoride concentrations have remained stable over the years, and the residents generally use a single source for their drinking water needs. Furthermore, due to limited 
mobility of the population, current exposures likely reflect chronic exposures.

Among possible confounders, both arsenic and lead are known to be low in drinking water in the area. Iron deficiency is known to impair motor and mental development in infants, children, and adolescents (CDC 2000), but the blood test results showed that the study children were not iron-deficient.

One of the strengths of our pilot study is that we were able to use multiple exposure biomarkers as indicators of the study children's fluoride exposures within a wide range of concentrations. Current well-water fluoride exposures were considered representative of chronic exposures. In addition, the urinary fluoride excretion after an exposure-free night was used as a reflection of the release from skeletal deposits of fluoride. Our findings suggest that among the biomarkers, dental fluorosis was most strongly related to children's test scores. A child's urinary fluoride concentration in a spot sample might be affected by the amount of bottled water that the child drank (if at all), and the current well-water fluoride concentrations could be imprecise as the internal dose also depends on the total water intake. The small numbers of children in the normal/questionable and mild/very mild Dean Index categories, resulting in low statistical power, may also have contributed to the null associations with outcomes in these two groups.

An additional strength of our study is the use of neurobehavioral tests that are thought to be relatively culture-independent and that reflect diverse functional domains, including memory and learning, attention and tracking, visuospatial organization and reasoning, motor and dexterity skills. Previous studies have employed only crude omnibus measures of neurobehavioral performance such as the Chinese Standardized Raven Test (rural version). The results suggest that the test battery is psychometrically appropriate in this population despite cultural differences 
from the samples on whom the tests were developed and normalized.

Our results showed that moderate and severe fluorosis was significantly associated with deficits in digit span scores. Digit span tests have been used to assess short-term memory and working memory. Deficits in digit span have been associated with methylmercury (Grandjean et al. 1997) and manganese exposures (Carvalho et al. 2013). The inverse association between fluoride and digit span suggests that the children's skills in auditory span or working memory may be particularly affected by elevated fluoride exposure. Digit span was the most sensitive test and appeared to be an appropriate test as the children were instructed to simply repeat strings of digits, thus requiring only a natural and habitual response from the subjects. This test should therefore be included in future studies to characterize the dose-response relationships and neuropsychological features of fluoride neurotoxicity.

Individual covariates were recorded and adjusted in the analysis. However, confounding bias appeared to be limited in this study as our population showed minimal diversity in their social characteristics. Background information on this community suggested that other neuropsychological risk factors were absent or would be unlikely to cause confounding.

It should be noted that in our study, the lowest fluoride concentration in well-water was 1.0 $\mathrm{mg} / \mathrm{L}$, a level that is within the current DHHS recommended level of fluoride in drinking water. The range of exposure levels was well within the ranges in other parts of the world, including Africa, the Eastern Mediterranean and southern Asia (India and Sri Lanka), Iraq, Iran, and northern China, where the highest fluoride concentration may reach $20 \mathrm{mg} / \mathrm{L}$ (WHO 2006). Apart from seasonal changes, the well-water fluoride concentrations have remained stable over the years, and the residents generally use the same source for their drinking water needs. Thus, 
no control group with minimal fluoride exposure was included.

Statistical power is also limited by the small size of the study population. However, in this perspective, the strong adverse association between dental fluorosis and the digit span outcome is noteworthy.

Results of our field study raise a concern about the safety of elevated systemic exposure to fluoride from high concentrations in the drinking water. While topical fluoride treatment confers benefits of reducing caries incidence, the systemic exposure should not be so high as to impair children's neurodevelopment especially during the highly vulnerable windows of brain development in utero and during infancy and childhood and may result in permanent brain injury. We are planning a larger scale study to better understand the dose-effect relationships for fluoride's developmental neurotoxicity in order to characterize the appropriate means of avoiding neurotoxic risks while securing oral health benefits.

\section{Acknowledgments}

This study was supported by internal institutional funds. We thank the parents, guardians, and children for their participation in this pilot study.

\section{Conflict of Interest}

None declared. 


\section{References}

Adams W, Sheslow D. Wide Range Assessment of Visual Motor Abilities. Wilmington, DE: Wide Range. 1995.

ATSDR (Agency for Toxic Substances and Disease Registry). Toxicological Profile for Fluorides, Hydrogen Fluoride, and Fluorine (Update). Atlanta, Ga: Agency for Toxic Substances and Disease Registry. 2003.

Carvalho CF, Menezes-Filho JA, Matos VP, Bessa JR, Coelho-Santos J, Viana GF, Argollo N, Abreu N. Elevated airborne manganese and low executive function in school-aged children in Brazil. Neurotoxicol 2013, http://dx.doi.org/10.1016/j.neuro.2013.11.006.

CDC (Center for Disease Control and Prevention). Morbidity and Mortality Weekly Report (MMWR) - Iron deficiency. 2000. 51:40. http://www.cdc.gov/mmwr/preview/mmwrhtml/mm5140a1.htm.

Chioca Lr, Raupp IM, Da Cunha C, Lossa EM, Andreatini R. Subchronic fluoride intake induces impairment in habituation and active avoidance tasks in rats. Eur J Pharmocol 2008;579:196201.

Choi AL, Sun GF, Zhang Y, Grandjean P. Developmental fluoride neurotoxicity: a systematic review and meta-analysis. Environ Health Perspect 2012;120:1362-1368.

Dean HT. The investigation of physiological effects by the epidemiological method. In: Moulton FR, ed. Fluorine and dental health. Washington: American Association for the Advancement of Science. 1942.

Dean HT, Elvove E. Some epidemiological aspects of chronic endemic dental fluorosis. Am J Public Health 1936;26:567-575.

Dobbling J. Vulnerable periods in developing brain. In: Davidson AN, Dobbing J, eds. Applied 
Neurochemistry. Philadelphia: Davis, pp.287-316. 1968.

Grandjean P, Weihe P, White RF, Debes F, Araki S, Yokoyama K, et al. Cognitive deficit in 7year-old children with prenatal exposure to methylmercury. Neurotoxicol Teratol. 1997;19:417-428.

Grandjean P, White RF, Nielsen A, Cleary D, de Oliveira Santos EC. Methylmercury neurotoxicity in Amazonian children downstream from gold mining. Environ Health Perspect 1999;107:587-591.

Grandjean P, Harari R, Barr DB, Debes F. Pesticide exposure and stunting as independent predictors of neurobehavioral deficits in Ecuadorian school children. Pediatr 2006;117:546556.

Grandjean P, Landrigan PJ. Developmental neurotoxicity of industrial chemicals. Lancet 2006;368:2167-2178.

Grandjean P, Landrigan PJ. Neurobehavioural effects of developmental toxicity. Lancet Neuro 2014;13:330-338.

Knights RM, Moule AD. Normative data on the motor steadiness battery for children. Perceptual and Motor Skills 1968; 26:643-650.

Lezak MD. Neuropsychological Assessment, $3^{\text {rd }}$ ed. Oxford: Oxford University Press. 1995.

Mullenix PJ, Denbesten PK, Schunior A, Kernan WJ. Neurotoxicity of sodoum fluoride in rats. Neurotoxicol Teratol 1995;17:169-177.

NRC (National Research Council). Fluoride in Drinking Water: A Scientific Review of EPA's Standards. Washington, DC:National Academies Press. 2006.

Rice D, Barone S Jr. Critical periods of vulnerability for the developing nervous system: 
evidence from humans and animal models. Environ Health Perspect 2000;108(suppl 3):511533.

Sheslow D, Adams W. Wide Range Assessment of Memory and Learning. Wilmington, DE: Jastak. 1990.

U.S. EPA. EPA and HHS Announce New Scientific Assessments and Actions on Fluoride: Agencies Working Together To Maintain Benefits of Preventing Tooth Decay while Preventing Excessive Exposure. Available:

http://yosemite.epa.gov/opa/admpress.nsf/6427a6b7538955c585257359003f0230/86964af5 77c37ab285257811005a8417!OpenDocument [accessed 18 February 2014].

Wechsler D. Wechsler Intelligence Scale for Children - Revised. New York, NY: Psychological Corp. 1974.

Whitford GM. The metabolism and toxicity of fluoride. $2^{\text {nd }}$ revised ed. New York: Karger. 1996. WHO (World Health Organization). Expert committee on water fluoridation. Technical Report No. 146. Geneva: World Health Organization. 1958.

WHO (World Health Organization). Oral health surveys. Basic methods. Geneva:World Health Organization. 1997.

WHO (World Health Organization). Fluorides. Geneva:World Health Organization. 2002.

WHO (World Health Organization). Fluoride in drinking water. Geneva: World Health Organization. 2006. 
Table 1. Characteristics of the 51 children (aged 6-8 years) in Mianning County

\begin{tabular}{|c|c|c|c|}
\hline Variable & $\begin{array}{c}\text { All } \\
(\mathrm{N}=51)\end{array}$ & $\begin{array}{c}\text { Boys } \\
(\mathrm{N}=24)\end{array}$ & $\begin{array}{c}\text { Girls } \\
(\mathrm{N}=27)\end{array}$ \\
\hline Age at examination (years) & $7.1(0.6)$ & $7.1(0.6)$ & $7.1(0.7)$ \\
\hline Sex (boys/girls) [\%] & $47 / 53$ & - & - \\
\hline Birth weight $(\mathrm{kg})^{\mathrm{a}}$ & $3.21(0.29)$ & $3.21(0.29)$ & $3.20(0.28)$ \\
\hline Weight at examination $(\mathrm{kg})^{\mathrm{a}}$ & $20.9(2.6)$ & $21.1(2.5)$ & $20.8(2.7)$ \\
\hline Height at examination $(\mathrm{m})^{\mathrm{a}}$ & $1.13(0.05)$ & $1.13(0.06)$ & $1.14(0.05)$ \\
\hline Systolic blood pressure $(\mathrm{mmHg})^{\mathrm{a}}$ & $81.9(6.0)$ & $82.5(5.1)$ & $81.4(6.8)$ \\
\hline Diastolic blood pressure $(\mathrm{mmHg})^{\mathrm{a}}$ & $60.0(5.9)$ & $61.0(5.5)$ & $59.1(6.2)$ \\
\hline Hemoglobin $(\mathrm{g} / \mathrm{dL})^{\mathrm{a}}$ & $11.8(1.0)$ & $12.0(0.8)$ & $11.6(1.1)$ \\
\hline Hemotocrit $(\%)^{\mathrm{a}}$ & $35(3.0)$ & $35.3(2.0)$ & $34.5(3.0)$ \\
\hline Parity $(1 / 2 / 3+)[\%]$ & $33 / 31 / 36$ & $38 / 25 / 37$ & $30 / 37 / 33$ \\
\hline Number in the household ${ }^{\mathrm{a}}$ & $6(2)$ & $6(2)$ & $6(2)$ \\
\hline $\begin{array}{l}\text { Household income in the past year (yuan): } \\
(<3000 / 3001-5000 />5000)[\%]\end{array}$ & $61 / 27 / 12$ & $50 / 33 / 17$ & $70 / 22 / 8$ \\
\hline \multicolumn{4}{|l|}{ Characteristics of parent/guardian: } \\
\hline Age (years) ${ }^{\mathrm{a}}$ & $35.7(7.8)$ & $34.9(4.2)$ & $36.3(9.9)$ \\
\hline $\begin{array}{l}\text { Relationship to child } \\
\text { (parents/grandparents/others) [\%] }\end{array}$ & $82 / 14 / 4$ & $86 / 4 / 8$ & $78 / 22 / 0$ \\
\hline $\begin{array}{l}\text { Education (elementary or below/ junior high } \\
\text { and above) [\%] }\end{array}$ & $27 / 73$ & $29 / 71$ & $26 / 74$ \\
\hline Occupation (farmers/others) [\%] & $96 / 4$ & $92 / 8$ & $100 / 0$ \\
\hline
\end{tabular}

${ }^{\mathrm{a}}$ Arithmetic mean (SD) 
Table 2. Distribution of Fluoride (F) Concentrations Among the 51 children in Mianning

\begin{tabular}{|c|c|c|c|c|}
\hline & $\begin{array}{c}\text { Geometric } \\
\text { mean }\end{array}$ & $\begin{array}{c}\text { Interquartile range } \\
\text { (IQR) }\end{array}$ & $\begin{array}{l}\text { Total } \\
\text { range }\end{array}$ & $\begin{array}{c}\text { Correlation } \\
\text { with F in } \\
\text { urine }\end{array}$ \\
\hline \multicolumn{5}{|l|}{ Fluoride } \\
\hline \multicolumn{5}{|l|}{ Concentrations: } \\
\hline Urine (mg/L) & 1.64 & $1.10-2.64$ & $0.22-5.84$ & (1) \\
\hline Water (mg/L) & 2.20 & $1.57-3.63$ & $1.0-4.07$ & 0.66 \\
\hline \multicolumn{5}{|l|}{ Dean Index ${ }^{a}(\%)^{b}$ : } \\
\hline \multicolumn{5}{|c|}{ Normal/Questionable (19\%) } \\
\hline Urine $F(\mathrm{mg} / \mathrm{L})$ & 0.45 & $0.37-0.61$ & $0.22-1.02$ & (1) \\
\hline Water F $(\mathrm{mg} / \mathrm{L})$ & $1.0^{\mathrm{c}}$ & - & - & - \\
\hline \multicolumn{5}{|c|}{ Very mild/Mild (21\%) } \\
\hline Urine F (mg/L) & 1.91 & $1.70-2.44$ & $0.34-4.78$ & (1) \\
\hline Water F $(\mathrm{mg} / \mathrm{L})$ & 2.10 & $1.57-3.37$ & $1.00-4.07$ & 0.79 \\
\hline \multicolumn{5}{|c|}{ Moderate/Severe $(60 \%)$} \\
\hline Urine F $(\mathrm{mg} / \mathrm{L})$ & 2.44 & $1.92-2.97$ & $1.10-5.84$ & (1) \\
\hline Water F $(\mathrm{mg} / \mathrm{L})$ & 2.66 & $2.13-3.63$ & $1.12-4.07$ & 0.12 \\
\hline
\end{tabular}

${ }^{a}$ Diagnosis on severity of fluorosis

${ }^{b}$ percent $(\%)$ of children in each category

${ }^{c}$ Fluoride concentration in well-water was $1.0 \mathrm{mg} / \mathrm{L}$ for all the subjects in this category 
Table 3. Distributions of neuropsychological tests among the 51 children

\begin{tabular}{|c|c|c|c|}
\hline Tests & Mean (SD) & $\begin{array}{c}\text { Interquartile } \\
\text { Range }\end{array}$ & $\begin{array}{r}\text { Total } \\
\text { Range } \\
\end{array}$ \\
\hline \multicolumn{4}{|l|}{ WRAML } \\
\hline Finger Windows & $9.96(3.1)$ & $8-12$ & $2-16$ \\
\hline $\begin{array}{l}\text { Visual Learning Total } \\
\text { (Trials 1-4) }\end{array}$ & $13.3(6.7)$ & $8-16$ & $0-32$ \\
\hline Visual Learning Delay & $4.4(2.7)$ & $3-6$ & $0-11$ \\
\hline $\begin{array}{l}\text { Visual Learning Difference } \\
\text { (Trials 1-4 Delay) }\end{array}$ & $-0.27(2.0)$ & $-1.5-1.0$ & $-5.0-5.0$ \\
\hline Design Memory & $18.9(6.0)$ & $14-22.5$ & $9-37$ \\
\hline \multicolumn{4}{|l|}{ WISC-R } \\
\hline $\begin{array}{l}\text { Squareroot Block Design } \\
\text { Digit Span }\end{array}$ & $2.62(1.1)$ & Digit Span & $0-4.6$ \\
\hline Forward & $8.2(1.8)$ & $7-10$ & $5-13$ \\
\hline Backward & $2.5(1.6)$ & $2-4$ & $0-6$ \\
\hline Total & $10.7(2.7)$ & $9-12$ & $5-18$ \\
\hline WRAVMA Drawing & $12.9(2.5)$ & $11-14$ & $6-19$ \\
\hline \multicolumn{4}{|l|}{$\begin{array}{l}\text { Finger Tapping (maximum in } \\
15 \mathrm{~s} \text { ) }\end{array}$} \\
\hline Preferred Hand & $30.6(5.0)$ & $27-35$ & $21-42$ \\
\hline Non-Preferred Hand & $28.0(4.2)$ & $25-31$ & $19-37$ \\
\hline \multicolumn{4}{|l|}{ Grooved Pegboard (s) } \\
\hline Log10 Dominant hand & $2.0(0.1)$ & $1.92-2.06$ & $1.80-2.32$ \\
\hline Log10 Non-dominant hand & $2.1(0.1)$ & $1.99-2.11$ & $1.87-2.30$ \\
\hline
\end{tabular}


Table 4. Adjusted effects ${ }^{\mathrm{a}}$ (betas and $95 \%$ confidence intervals) of fluoride concentrations on the neuropsychological tests among the 51 children

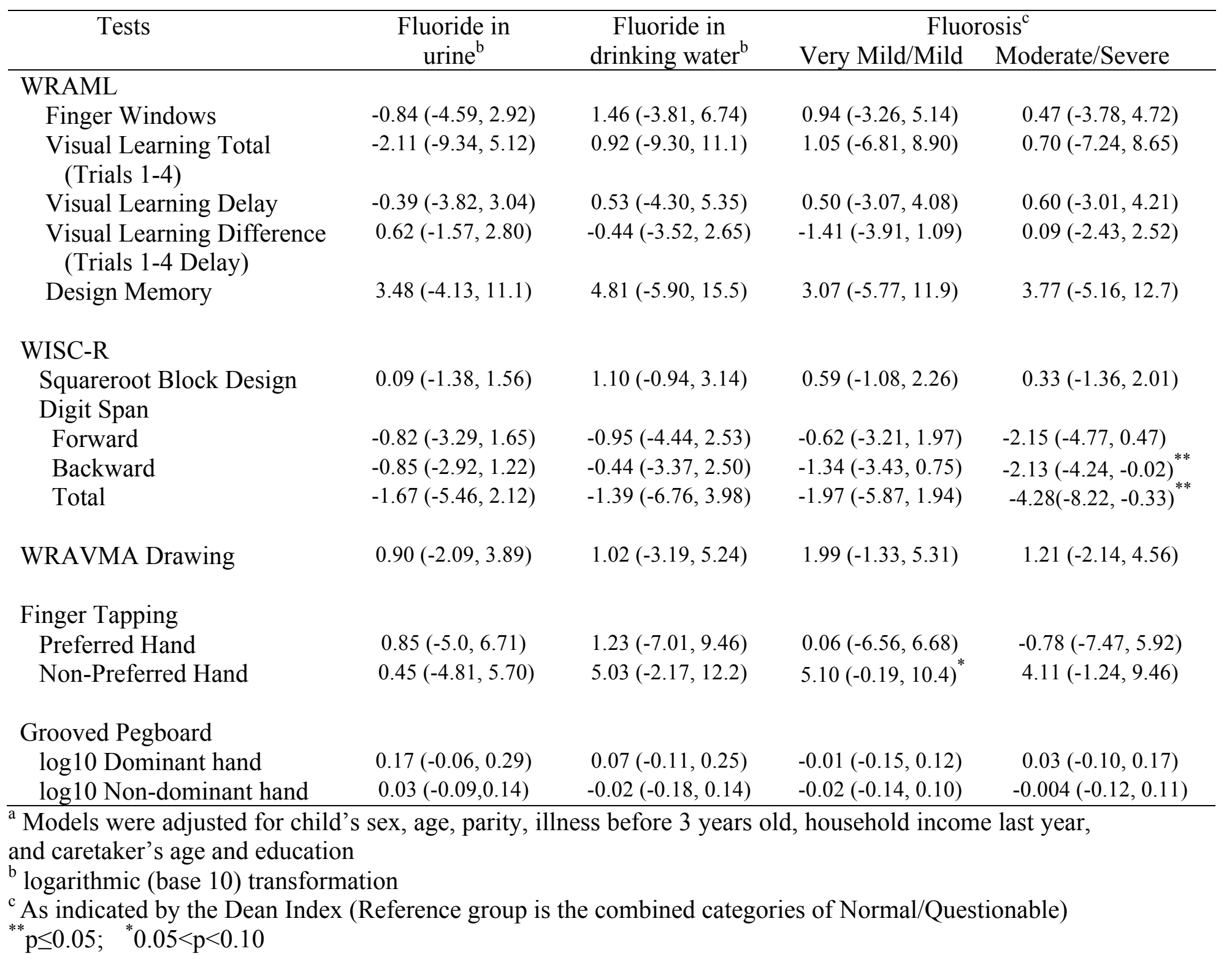


Table 5. Adjusted means ${ }^{\mathrm{a}}(95 \%$ confidence intervals) of the neuropsychological tests by Dean Index among the 51 children

\begin{tabular}{|c|c|c|c|}
\hline \multirow[t]{2}{*}{ Tests } & \multicolumn{3}{|c|}{ Fluorosis $^{b}$} \\
\hline & $\begin{array}{l}\text { Normal/Questionable } \\
\qquad \mathrm{N}=8\end{array}$ & $\begin{array}{l}\text { Very Mild/Mild } \\
\qquad \mathrm{N}=9\end{array}$ & $\begin{array}{l}\text { Moderate/Severe } \\
\quad \mathrm{N}=26\end{array}$ \\
\hline \multicolumn{4}{|l|}{ WRAML } \\
\hline Finger Windows & $9.48(6.71,12.2)$ & $10.2(7.53,12.9)$ & $9.76(7.44,12.1)$ \\
\hline $\begin{array}{l}\text { Visual Learning Total } \\
\text { (Trials 1-4) }\end{array}$ & $10.1(4.96,15.3)$ & $11.5(6.49,16.6)$ & $11.2(6.86,15.5)$ \\
\hline Visual Learning Delay & $3.20(0.85,5.54)$ & $3.90(1.60,6.20)$ & $4.0(2.02,6.0)$ \\
\hline $\begin{array}{l}\text { Visual Learning Difference } \\
\text { (Trials 1-4 Delay) }\end{array}$ & $-0.45(-2.11,1.22)$ & $-1.64(-3.25,-0.03)$ & $-0.13(-1.51,1.25)$ \\
\hline Design Memory & $16.4(10.6,22.2)$ & $14.1(13.9,25.2)$ & $20.2(15.4,25.1)$ \\
\hline \multicolumn{4}{|l|}{ WISC-R } \\
\hline Squareroot Block Design & $2.73(1.63,3.83)$ & $2.81(1.74,3.88)$ & $2.54(1.62,3.46)$ \\
\hline \multicolumn{4}{|l|}{ Digit Span } \\
\hline Forward & $9.61(7.92,11.3)^{* \mathrm{c}, \mathrm{d}}$ & $8.76(7.09,10.4)^{*_{\mathrm{c}}}$ & $7.23(5.81,8.67)$ \\
\hline Backward & $3.23(1.86,4.61)^{* d}$ & $1.86(0.51,3.20)$ & $1.07(-0.08,2.22)^{* * \mathrm{~d}}$ \\
\hline Total & $12.8(10.3,15.4)^{*_{c}, * *_{\mathrm{e}}}$ & $10.6(8.11,13.1)^{*_{c}}$ & $8.31(6.15,10.5)^{* *_{e}}$ \\
\hline WRAMA Drawing & $12.1(9.91,14.3)$ & $14.1(1.9,16.2)$ & $13.3(11.5,15.1)$ \\
\hline \multicolumn{4}{|l|}{ Finger Tapping } \\
\hline Preferred Hand & $29.5(25.3,33.6)$ & $29.5(25.3,33.8)$ & $28.7(25.0,32.3)$ \\
\hline Non-Preferred Hand & $24.7(21.3,28.0)$ & $29.8(26.4,33.2)$ & $28.8(25.8,31.7)$ \\
\hline \multicolumn{4}{|l|}{ Grooved Pegboard } \\
\hline $\log 10$ Dominant hand & $1.97(1.88,2.06)$ & $1.98(1.89,2.06)$ & $2.02(1.95,2.10)$ \\
\hline $\log 10$ Non-dominant hand & $2.07(2.0,2.15)$ & $2.04(1.97,2.12)$ & $2.06(1.99,2.12)$ \\
\hline \multirow{2}{*}{\multicolumn{4}{|c|}{$\begin{array}{l}\text { Adjusted for child's sex, age, parity, illness before } 3 \text { years old, household income last year, and } \\
\text { caretaker's age and education } \\
\text { b As indicated by the Dean Index }\end{array}$}} \\
\hline & & & \\
\hline \multicolumn{4}{|c|}{$\begin{array}{l}{ }^{c} \text { "Significant mean difference between the } 2 \text { categories: Normal/Questionable and Very Mild/Mild } \\
\text { at } 0.5<p<0.10\end{array}$} \\
\hline \multicolumn{4}{|c|}{$\begin{array}{l}\text { d Significant mean difference between the } 2 \text { categories: Normal/Questionable and Moderate/Severe } \\
\text { at } 0.5<p<0.10\end{array}$} \\
\hline \multicolumn{4}{|c|}{$\begin{array}{l}{ }^{* *} \text { Significant mean difference between the } 2 \text { categories: Normal/Questionable and } \\
\text { Moderate/Severe at } p<0.05\end{array}$} \\
\hline
\end{tabular}

